\title{
Observatorio Medioambiental
} ISSN: 1139-1987

http://dx.doi.org/10.5209/OBMD.54168

\section{Manejo y separación de residuos sólidos urbanos. Análisis comparativo entre Madrid (España) y el distrito especial industrial y portuario de Barranquillas (Colombia)}

\author{
Norida Suley Freiles Ariza \\ Recibido: 8 de junio del 2016/ Enviado a evaluar: 10 de junio del 2016/ Aceptado: 25 de octubre del 2016
}

\begin{abstract}
Resumen. El incremento de la generación de residuos sólidos es un problema que afecta con un notable deterioro al medio ambiente. Un buen manejo y aprovechamiento de los mismos es crucial para mejorar la calidad de vida en las ciudades. En el presente trabajo se analizan comparativamente la gestión de los residuos sólidos urbanos en las ciudades de Barranquilla y Madrid. Para obtener la información se han consultado diferentes fuentes desde páginas web municipales, a documentos de prensa, pasando por revistas científicas, artículos de divulgación, entrevistas a técnicos responsables y visitas a centros de tratamiento. Los resultados muestran notables diferencias entre los dos municipios analizados, observando la importancia de la normativa legal, las infraestructuras que permiten su desarrollo y la concienciación ciudadana adquirida en programas de educación ambiental como factores ligados que contribuyen decisivamente al éxito en la gestión de los residuos sólidos urbanos.
\end{abstract}

Palabras clave: Manejo y separación de RSU; normas; educación ambiental.

\section{[en] Management and separation of urban solid waste comparative analysis between Madrid (Spain) and the industrial and port special district of Barranquilla (Colombia)}

\begin{abstract}
The increase in solid waste generation is a problem affecting a noticeable deterioration of the environment. Good handling and use of them is crucial to improve the quality of life in cities. In this paper comparatively analyzed the management of municipal solid waste in the cities of Barranquilla and Madrid. For information were consulted various sources from municipal web pages, documents press, through scientific journals, popular articles, interviews with technical experts and visits to treatment centers. The results show significant differences between the two municipalities analyzed, noting the importance of the legal regulations, infrastructures that allow development and public awareness acquired in environmental education programs and related factors contributing decisively to the successful management of solid waste urban.
\end{abstract}

Key words: Management and separation of municipal solid waste; standards; environmental education. 
[fr] Manipulation et separation der dèchets solides analyse comparative de Madrid (Espagne) et extraordinaire industrial district et port de Barranquillas (Colombia)

Résumé. L'augmentation de la production de déchets solides est un problème affectant une détérioration notable de l'environnement. Bonne manipulation et l'utilisation d'entre eux est essentielle pour améliorer la qualité de vie dans les villes. Dans cet article, relativement analysé la gestion des déchets solides municipaux dans les villes de Barranquilla et Madrid. Pour plus d'informations ont été consultées diverses sources de pages Web municipaux, documents de presse, par le biais de revues scientifiques, des articles populaires, des interviews avec des experts techniques et des visites aux centres de traitement. Les résultats montrent des différences significatives entre les deux communes analysées, en notant l'importance de la réglementation légale, les infrastructures qui permettent le développement et la sensibilisation du public acquis dans les programmes d'éducation à l'environnement et les facteurs connexes qui contribuent de manière décisive à la bonne gestion des déchets solides urbain.

Mots clés: Gestion RSU et de séparation; normes; éducation à l'environnement.

Cómo citar. Freiles Ariza, N.S. (2016): Manejo y separación de residuos sólidos urbanos. Análisis comparativo entre Madrid (España) y el distrito especial industrial y portuario de Barranquilla (Colombia). Observatorio Medioambiental, 19, 197-211.

Sumario. 1. Introducción. 2. Localización y descripción de las areas de estudio. 3. La importancia de la Educación Ambiental. 3.1. Actividades de Educación Ambiental sobre el manejo y separación de RSU en el Distrito Industrial y Portuario de Barranquilla. 3.2. Actividades de Educación Ambiental sobre el manejo y separación de RSU en la ciudad de Madrid. 4. Marco legal y gestión de los Residuos Sólidos Urbanos (RSU). 4.1. Normativa y Planes de Gestión en el Distrito Especial, Industrial y Portuario de Barranquilla. 4.2. Normativa y Planes de Gestión en la ciudad de Madrid. 5. Conclusión. 6. Bibliografía.

\section{Introducción}

Los residuos existen desde que nuestro planeta tiene seres vivos, hace unos 4.000 millones de años. Antiguamente, la eliminación de los residuos humanos no planteaba un problema significativo, ya que la población era pequeña y la cantidad de terreno disponible para la asimilación de los residuos era grande. Sin embargo, la problemática de los residuos comienza con el desarrollo de la sociedad moderna en la que vivimos, no sólo en el aspecto referido a la cantidad de residuos que ésta genera (difícilmente asimilable por la naturaleza), sino, y de manera importantísima, a la calidad de los mismos. Este problema de la gestión de los residuos existe y se agrava año tras año y puede señalarse que cuatro son sus causas principales: En concreto, el rápido crecimiento demográfico, La concentración de la población en los centros urbanos, la utilización de bienes materiales de rápido deterioro y el uso cada vez más generalizado de envases sin retorno, fabricados con materiales no degradables.

Son varias las definiciones que se pueden encontrar del término "residuo". La Organización para la Cooperación y el Desarrollo Económico (OCDE) los define como "aquellas materias que, generadas en las actividades de producción y consumo, no alcanzan en el contexto en el que se producen ningún valor económico, bien porque no existe una tecnología adecuada para su aprovechamiento o bien porque no 
existe un mercado para los productos recuperados".Por otro lado, los instrumentos económicos basados en el incentivo son aquellos que permiten conseguir unos objetivos prefijados favoreciendo o desfavoreciendo (en general, económicamente) algunas conductas de los agentes del mercado. Así, el cambio de las actitudes sobre las que se quiere incidir se produce no como respuesta a unas normas de obligado cumplimiento, sino porque los sujetos a quienes va dirigido adoptan voluntariamente acciones que conducen a la consecución de los objetivos fijados por la administración, puesto que estas acciones pasan a ser económicamente más rentables como consecuencia de los incentivos. En este sentido, para que una buena gestión de los RSU funcione, se requiere la acción conjunta de dos factores complementarios, por una parte, la normativa que regule y eventualmente permita sancionar a quien la incumpla y por otra, la educación ambiental que modifique las conductas de la ciudadanía. Este último aspecto se va a tratar en el presente trabajo, analizando comparativamente lo que sucede en Madrid y Barranquilla.El objetivo es, Analizar cómo se efectúa el manejo y separación de los residuos sólidos urbanos en el Distrito Especial Industrial y Portuario de Barranquilla estableciendo una comparación con la estrategia seguida en las áreas urbanas de la ciudad de Madrid. Así mismo, evaluar la importancia de la cultura y educación ambiental en el problema planteado, a través de la información sobre los hábitos y actitudes de los habitantes de ambos municipios. Todo ello, con la finalidad de señalar cuáles son las principales debilidades del sistema de gestión en la primera y establecer recomendaciones para mejorarlo.

\section{Localización y descripción de las areas de estudio}

Barranquilla oficialmente Distrito Especial, Industrial y Portuario, está localizada en el vértice nororiental del departamento del Atlántico, sobre la orilla occidental del río Magdalena, a 7,5 km de su desembocadura en el mar Caribe. La población de Barranquilla es de 1.112.889 personas según el censo general del Departamento Administrativo Nacional de Estadística DANE del 2005, lo que la convierte en la cuarta ciudad más poblada del país detrás de Bogotá, Medellín y Cali. La ciudad es el núcleo del Área Metropolitana de Barranquilla, la cual está constituida además por los municipios de Soledad, Malambo, Galapa y Puerto Colombia.

El conglomerado alberga a 1.726.271 habitantes según el censo general del DANE del 2005, y ocupa también la cuarta posición entre las conurbaciones del país. De acuerdo con Ley 768 de 2002,76 el distrito de Barranquilla está dividido política y administrativamente en cinco localidades: Riomar, Norte-Centro Histórico, Sur Occidente, Metropolitana y Sur Oriente.El clima de la ciudad de Barranquilla es de tipo tropical seco, correspondiente a una vegetación propia de la sequedad y con una temperatura promedio de $27^{\circ} \mathrm{C}$. Barranquilla no tiene estaciones pero cuenta con dos períodos en el año: un período seco y otro de lluviasPor otro lado la ciudad de Madrid constituye el territorio uniprovincial de la Comunidad Autónoma de Madrid. La ciudad de Madrid se encuentra en la zona central de la península ibérica, a pocos 
kilómetros al norte del cerro de los Ángeles, centro geográfico de esta. La ciudad de Madrid constituye una metrópoli con 3.141.991 habitantes empadronados según datos del INE al 1 de enero del 2015. Cabe señalar, la cifra de población asciende a 6.436.996 habitantes en toda la comunidad según datos del INE al 1 de enero del 2015, referente del desarrollo económico - social de España. Madrid está dividido administrativamente en 21 distritos, que a su vez se subdividen en barrios, no necesariamente coincidentes con los barrios tradicionales. En total hay 21 distritos y 128 barrios. El clima es templado y cálido en Madrid. En invierno hay en Madrid mucho más lluvia que en verano. La clasificación del clima de Köppen-Geiger es Csa (Clima mediterráneo).La temperatura media anual en Madrid se encuentra a $13.7^{\circ} \mathrm{C}$.

En este sentido, el presente trabajo se ha efectuado obteniendo información procedente de diferentes fuentes, tanto a partir de entrevistas y trabajo de campo como de la consulta de las páginas correspondientes a organismos oficiales y empresas. Todo ello, complementado con una revisión bibliográfica procedente libros, de revistas científicas y otros documentos periodísticos o de divulgación, adicionalmente entrevistas con doctores relacionados al tema.

\section{La importancia de la Educación Ambiental}

La necesidad de una Educación relativa al Medio Ambiente o Educación Ambiental (EA) ha estado reconocida por la comunidad internacional desde la Conferencia de las Naciones Unidas Celebrada en Estocolmo (1972) sobre el medio ambiente. Desde esta fecha histórica para la E.A., han ido sucediéndose importantes reuniones nacionales e internacionales: Coloquio de Belgrado (1975), Conferencia de Tbilisi (1977), Conferencia de Moscú (1987), así como numerosos encuentros, Seminarios y Jornadas de carácter autonómico, provincial o local. Todas estas reuniones han ido configurando esta modalidad educativa. De todos estos encuentros fue la Conferencia de Tbilisi la que cuestionó de manera global las finalidades de la EA y que considera como los fines explícitos de todo programa de la EA.A continuación se analizan las actividades de EA relacionadas al manejo y separación de residuos sólidos urbanos en el Distrito de Barranquilla y la Ciudad de Madrid, teniendo en cuenta que en el Distrito los residuos son denominados residuos sólidos, desglosados a su vez en ordinarios y especiales. Por otra parte en la ciudad de Madrid y de acuerdo a la ordenanza de limpieza son denominados en residuos urbanos o municipales y estos a su vez se dividen es generales y especiales.

\subsection{Actividades de Ecuación Ambiental sobre el manejo y separación de RSU en el Distrito Industrial y Portuario de Barranquilla}

La empresa privada encargada de realizar el servicio de aseo en el Distrito Especial, Industrial y Portuario de Barranquilla es la Sociedad de Acueducto, Alcantarillado y Aseo de Barranquilla S.A. E.S.P. (Triple A), como empresa dedicada a la operación de los servicios mencionados, tiene su ámbito de operación en Colombia, 
específicamente en 15 municipios del departamento del Atlántico: entre ellos Barranquilla (Distrito). Su sede principal está ubicada en Barranquilla. Triple A ha liderado por 7 años la estrategia de cultura ciudadana Vive Barranquilla Limpia, logrando significativos cambios en los comportamientos de los barranquilleros frente al tema de la limpieza y el aseo de la ciudad. La estrategia Vive Barranquilla Limpia surgió como respuesta al compromiso de la Sociedad de Acueducto, Alcantarillado y Aseo de Barranquilla, Triple A, de emprender acciones orientadas al bienestar de la ciudad fundamentadas en procesos de educación ciudadana. Fue concebida como una estrategia de cultura ciudadana enfocada al logro de comportamientos responsables frente al aseo de la ciudad contribuyendo así a la gestión sostenible de los residuos. Dicha campaña responde al objetivo estratégico corporativo de contribuir con la competitividad de la ciudad a partir de la construcción de un entorno limpio apoyándose en los programas: Mi colegio Limpio. Mi barrio sin basuras. La cuadra ejemplar, y Limpiemos a Barranquilla en una hora.

A través del programa de Mi barrio sin basura, se desarrolló la estrategia de participación y compromiso ciudadano frente al aseo de la ciudad, activado en forma de concurso barrial orientado a crear sentido de pertenencia por la ciudad y mejorar el entorno. Por otra parte, Vive Barranquilla Limpia trabaja en los colegios para inculcar en los estudiantes la responsabilidad personal con el aseo de la ciudad, fomentando hábitos de cultura ciudadana a partir de sus referentes inmediatos: colegio, familia, barrios. Otro programa ya citado es limpiemos a barranquilla en una hora, se trata de una convocatoria masiva invitando a los barranquilleros y a todos los residentes de la ciudad para hacer un alto en sus jornadas, un día establecido a una hora establecida, con escoba y otros elementos de limpieza barriendo las calles de la ciudad, los frentes de casas, colegios, universidades y empresas. Así también está el programa Lacuadra ejemplar es una propuesta de ciudad en la que a través de un trabajo en equipo entre la comunidad y Triple A, se construye un escenario ejemplar y modelo en la prestación del servicio de aseo a partir del cumplimiento de actuaciones ciudadanas responsables, este programa solo se realizó en el año 2011.Por otro lado en año 2012, Triple A lanza el programa, denominado VerdeAzul 'Separarnos une', busca generar una nueva cultura en el ámbito de operación de la empresa y en toda la ciudadanía. El programa si bien se fundamenta en la responsabilidad hacia el medio ambiente, contempla hacia los usuarios dos tipos de retorno, denominado $2 \mathrm{R}$ : reconocimiento y remuneración.A partir del 2015, Triple A evoluciona su estrategia de cultura ciudadana: Vive Barranquilla Limpia pasó a llamarse Vive tu Ciudad Limpia. En él mes de febrero Triple A abrió convocatoria escolar para Mi colegio limpio 2016. El programa continua la experiencia de reciclaje escolar con material plástico Pet tipo1, manejo de residuos sólidos y actividades ecológicas. Por otro lado, el Distrito de Barranquilla está a cargo del DAMAB, como autoridad ambiental y rector de la política ambiental en materia de control y vigilancia. Más sin embargo a Barranquilla no le ha ido nada bien desde que fue expedida la Ley General Ambiental de Colombia (Ley 99 de 1993) y comenzó el proceso de descentralización de este sector a nivel territorial. Doce años después de creado el DAMAB, el 'fantasma' de la liquidación 
lo está rondando. En Barranquilla, uno de los factores más contaminantes es la mala disposición de los residuos sólidos, evidentemente existe una falta de cultura ciudadana que se ve evidenciada en las calles de la ciudad, las esquinas, andenes y zonas urbanas que se han convertido en botaderos informales. El problema de más incidencia en la ciudad es el de los botaderos a cielo abierto, en los que no se logra una total erradicación debido a que la gente no responde a las campañas realizadas por las diferentes entidades que regulan el espacio público y el medio ambiente en la ciudad.

\subsection{Actividades de Educación Ambiental sobre el manejo y separación de RSU en la ciudad de Madrid}

Consciente de las dudas que los ciudadanos y ciudadanas tienen acerca de cómo separar los residuos domésticos, el Ayuntamiento de Madrid, a través del Área de Gobierno de Medio Ambiente y Movilidad, realizo desde el año 2009 al 2012 la campaña Para no dudar al separar. Dicha campaña, se efectuó en colaboración con la empresa Ecoembes. Además, ha publicado la "Guía para resolver dudas sobre la separación de residuos domésticos en Madrid" y su versión infantil "Guía para aprender a separar residuos domésticos en Madrid (para niños y niñas de 7 a 11 años)", todo ello con el fin de responder a las preguntas recibidas desde que se implantó el sistema actual de separación de basuras en la ciudad. Actualmente se llama "Guía para resolver dudas sobre la separación de residuos en Madrid" (eliminando la palabra domésticos) e igualmente incluye las denominadas "3R": reducir, reutilizar y reciclar los residuos". Adicionalmente el Ayuntamiento ha editado las "Guías de buenas prácticas para reducir los residuos urbanos" así como otros folletos sobre el tema destinados a padres y profesores sobre cómo reducir, reutilizar y reciclar para un consumo sostenible.

Estas intervenciones se han llevado a cabo cronológicamente tal y como se reporta a continuación: En 2009-2010: desde el 21 de diciembre de 2009 al 14 de mayo de 2010. En los 21 distritos de Madrid. En cada distrito se ha actuado durante tres semanas, en 2010- 2011 entre los meses 10 de enero al 2 de junio de 2011 en los en los 21 distritos de Madrid. En cada distrito se ha actuado durante una semana".

La tercera campaña de separación de residuos 'Para no dudar al separar" se ha desarrollado entre diciembre de 2011 y abril de 2012 en más de 30 centros de la Universidad Complutense de Madrid y de la Universidad Nacional de Educación a Distancia.En la campaña informativa sobre separación se realizaron tres tipos de intervenciones, Actuaciones en el barrio, actuaciones en el colegio/os, actuaciones realizadas en otros Centros y Asociaciones. Con el objetivos de Sensibilizar a los ciudadanos/as y resolver sus dudas acerca de la separación de residuos domésticos y correcta disposición en los diferentes contenedores entre otros objetivos relacionados a ello.En la actualidad el Ayuntamiento de Madrid a través de la Dirección General del Parque Tecnológico de Valdemingómez, dispone de un programa de EA. Programa de Educación Ambiental Integral, que constituye una referencia a nivel nacional e internacional, no solo por el número de visitantes (más de 11.000 al año), 
sino porque concentra en un solo recinto todos los procesos industriales que, hoy día, se pueden realizar con los residuos, constituyendo unas instalaciones únicas desde un punto de vista técnico y didáctico. Las actuaciones incluidas en dicho programa, en marcha desde septiembre de 2007, contemplan visitas al Parque adaptadas a la capacidad de comprensión, aprendizaje e interés de los diferentes grupos de población que pueden participar en ellas. A este respecto, los grupos considerados para el diseño de las visitas han sido los siguientes como, Escolares de 6 a 12 años, Escolares de 12 a 16 años, Universitarios, Postgraduados, Profesionales del sector, Visitas institucionales y público en general. La superficie destinada a funciones educativas, asciende a $1.925 \mathrm{~m}^{2}$, distribuidos en seis edificios: Centro de Visitantes, Aula Infantil, Centro Las Dehesas, Centro La Paloma, Centro La Galiana, Centro Las Lomas. A continuación se mencionan algunas de las compañas más recientes.

La campaña para el fomento de la separación de residuos (2016) Separa, Recicla, Quiere a Madrid. Como parte de la campaña de sensibilización y concienciación ambiental que en materia de limpieza y cuidado de espacios públicos llevo a cabo el Ayuntamiento de Madrid en sus 21 distritos, el Área de Gobierno de Medio Ambiente y Movilidad, en colaboración con Ecoembes, puso en marcha una Campaña específica para fomentar y mejorar la separación selectiva de residuos domiciliarios con el lema Separa, Recicla, Quiere a Madrid. De forma paralela a esta campaña de residuos, en el mes de febrero del presente año 2016 se lanzó la segunda fase de la campaña de sensibilización general que inició el Ayuntamiento el pasado mes de octubre del año 2015, para incrementar la colaboración de la ciudadanía en el mantenimiento de la limpieza de la ciudad. La publicación del bando de la Alcaldesa de Madrid marca el comienzo de la segunda fase. La campaña de sensibilización incluye diversas acciones como, entre otras, el reparto de ceniceros de bolsillo, acciones pedagógicas en el tejido comercial de los veintiún distritos o en centros escolares, la colocación de señales permanentes en todos los barrios de Madrid o la difusión de mensajes a través de medios digitales y soportes de publicidad exterior. Esta campaña de sensibilización se desarrollará lo largo de 2 años. Sobre los 25000 euros de gasto inicial correspondientes al diseño de toda la campaña, incluyendo gráficas y primer video informativo.

Por otra parte, el programa Educar hoy por un Madrid más sostenible es una actividad de educación ambiental del Ayuntamiento de Madrid, cuyo objeto es mejorar el medio ambiente escolar, local y global, a fin de hacer de Madrid un ecosistema urbano sostenible y una ciudad con calidad de vida. En este sentido, la Cumbre sobre el Cambio Climático (COP21; Paris 2015), señala el papel de la educación para el desarrollo sostenible en el logro de la sostenibilidad de las ciudades y del planeta. El reto es poner en manos de los estudiantes la posibilidad de actuar responsablemente, a través de proyectos e iniciativas que favorezcan su participación en la solución de los problemas ambientales.Adicionalmente, la acreditación Centro Ambientalmente Sostenible es un reconocimiento que el Ayuntamiento de Madrid otorga, en el marco del programa "Educar hoy por un Madrid más sostenible", a los centros docentes que desarrollan proyectos de educación ambiental que mejoran el 
entorno escolar y contribuyen a hacer de Madrid una ciudad con calidad de vida. Se han establecido tres niveles de acreditación o calidad (de mayor a menor grado de sostenibilidad): Nivel I (verde), Nivel II (azul) y Nivel III (amarillo),en función de los retos, objetivos y logros alcanzados. Asimismo España cuenta con del Libro Blanco de la Educación Ambiental. El Libro Blanco ofrece un mosaico de ideas, sugerencias y propuestas para que la educación ambiental contribuya a mejorar la participación de la población en la prevención y resolución de los problemas ambientales.

\section{Marco legal y gestión de los Residuos Sólidos Urbanos (RSU)}

El manejo y separación de los RSU se desenvuelve en un marco legal amplio y más o menos estricto en el que los diferentes estados desarrollan planes de gestión que complementan la educación ambiental con un cometido eventualmente sancionador. Como se ha indicado, la mayor eficacia puede conseguirse combinando estrategias educativas y de sensibilización con normas disuasorias que impliquen el pago de multas u otras circunstancias de coacción. A continuación un análisis de ambas ciudades.

\subsection{Normativa y Planes de Gestión en el Distrito Especial, Industrial y Portuario de Barranquilla}

En este sentido el Distrito Especial, Industrial y Portuario de Barranquilla dispone del Plan de Gestión Integral de Residuos sólidos 2016- 2027. Este documento aborda los aspectos más importantes para el logro del cambio cultural que se busca en los habitantes del Distrito, que va desde la minimización y clasificación de residuos en origen hasta la su valorización, aprovechamiento y disposición final.Dentro de este Plan de Gestión Integral de Residuos sólidos se dispone un marco legal, el cual está constituido primeramente de carácter general, segundo por servicio público de aseo y por ultimo a nivel Distrital, en conjunto de estos tres segmentos encontramos 3 Leyes, 2 Resoluciones, 4 Decretos y la Constituciones Colombiana de 1991, artículos 365 al 370. Todas ellas relacionas a residuos sólidos.

Citado anteriormente el Distrito Especial, Industrial y Portuario de Barranquilla dispone de un Plan de Gestión Integral de Residuos Sólidos - PGIRS 2016 - 2027. A efectos de este PGIRS lo residuos sólidos se definen como cualquier objeto, material, sustancia o elemento principalmente sólido resultante del consumo o uso de un bien en actividades domésticas, industriales, comerciales, institucionales o de servicios, que el generador presenta para su recolección por parte de la persona prestadora del servicio público de aseo. Igualmente, se considera como residuo sólido, aquel proveniente del barrido y limpieza de áreas y vías públicas, corte de césped y poda de árboles. Estos se dividen en, residuo sólido ordinario y residuo sólido especial. Los residuos sólidos que no tienen características de peligrosidad se dividen en aprovechables y no aprovechables. 
El servicio de gestión de aseo, está a cargo de la sociedad mencionada. Este a su vez, realiza los procesos de Barrido, recolección domiciliaria, transporte y disposición final de residuos sólidos, hacen parte de este servicio. La empresa atiende también a usuarios no residenciales que producen un volumen superior al metro cúbico de residuos al mes, a través de modernos equipos de compactación. Actualmente el Distrito de Barranquilla no cuenta con un estudio de caracterización de residuos sólidos en la fuente para el área urbana. Más sin embargo, según el título F del RAS, establece un esquema de clasificación según la composición física teniendo en cuenta que los residuos sólidos deben clasificarse, en porcentaje en peso base húmeda, de acuerdo con el siguiente esquema: "Residuos orgánicos crudos, residuos de poda, corte de césped y jardinería, Productos de papel y productos de cartón, Plásticos, Textiles, Metales ferrosos, compuestos de aluminio y otros metales no ferrosos, Vidrio, Madera, caucho (goma), cuero, ceniza, rocas y escombros, huesos y otros".

En este sentido, en Barranquilla no existe un sistema de reciclaje a gran escala, como lo hay en otras ciudades, donde el usuario puede separar los desechos de su hogar en tres canecas de diferentes colores y la empresa de aseo convertirlos en material de reciclaje. No obstante, la empresa Triple A recomienda separar los desechos orgánicos (especialmente alimentos) de los llamados "reciclables", como latas, botellas y cartones. Es decir, lo que en el mundo tiene muchos caminos, debido a los planes de reciclaje, en Barranquilla sólo tiene dos. Los desechos orgánicos deben ser entregados al camión de la basura. Y los otros desechos, los "reciclables" locales, colocados en una caneca especial para que lo recojan los basureros de la calle. En la descripción del presente programa se partirá de las frecuencias y horarios actuales realizados por Triple A S.A. E.S.P.Por otra parte, actualmente la Triple A, presta a los usuarios el servicio de recolección de podas, troncos, escombros y otros (aserrín, madera, icopor, llantas, colchones y enseres); el cual consiste en que los usuarios solicitan el servicio, luego la empresa genera una cotización del servicio y si el usuario confirma la prestación del servicio los residuos son recogidos en un tiempo establecido y transportados hasta el Relleno Sanitario Los Pocitos. EL Distrito no cuenta con un estudio de caracterización de los residuos sólidos especiales generados. El barrido también hace parte de la dinámica de aseo. Existe el barrido mecánico, que se efectúa con la ayuda de una máquina barredora y equipos de alto rendimiento, con filtros anti-polvo y potente aspiración; y el barrido manual, que utiliza la mano de obra directa para sitios de difícil acceso y limpieza de las calles. Para el baldeo de vías se utiliza agua a alta presión para desincrustar la suciedad en aquellos sitios que requieren altos niveles de limpieza (Plazas y Parques).

$\mathrm{Y}$, finalmente, son transportados para su disposición final del residuo sólido, este se realiza en el Relleno Sanitario "Parque Ambiental Los Pocitos" surge como solución al manejo de los residuos sólidos del área metropolitana del Atlántico teniendo en cuenta que el antiguo relleno sanitario El Henequén llego a su capacidad máxima de disposición. El Relleno Sanitario Parque Ambiental Los Pocitos comenzó a operar en abril de 2009. Está localizado a 15 kilómetros de Barranquilla, en la vía Juan Mina-Tubará, sobre un área total de 135 hectáreas, de las cuales 75 hectáreas 
son para la disposición de las cerca de 1.300 toneladas diarias de desechos sólidos. De acuerdo con el Director de disposición final de residuos del relleno sanitario los pocitos Samir Guzmán Guzmán. Comenta que el proceso de descomposición normal de los residuos sólidos son dos. Uno que lixivian y otro que genera biogás, en este primer proceso de descomposición que es el lixiviado son los líquidos o las aguas que botan los residuos sólidos o basura en su proceso natural de descomposición y son reutilizados en el mismo parque los pocitos para su vertimiento. $\mathrm{Y}$ el biogás es transportado por medio de chimeneas internas dentro de los vasos. Triple A, ha desarrollado un proyecto MDL (Mecanismo de Desarrollo Limpio), el cual captura este biogás y lo quema a temperatura entre 800 y 1200 grados centígrados. Garantizando que se elimine en su totalidad el metano, al ser quemado este biogás se genera $\mathrm{CO}^{2}$, que es adsorbido por los árboles en su proceso natural de fotosíntesis impactando de manera positivamente al medio ambiente.

De acuerdo a la proyección de generación de residuos sólidosdel PGIRS, la cantidad de residuos sólidos generados por habitantes fue de 0,8950 , generación al día de 1.090.456,22 y generación al año398.016,52. Datos del año 2015 con una población de 1.218.387. En este sentido, esta proyección muestra que para el año 2028 con una población de 1.370 .625 , tendrá una generación por día de 1,0186 , una generación al día de 1.396.109,40, y al año de 509.579,93, y toneladas acumuladas de 6.323.518,43. De manera queda establecida la generación del año 2028, donde se tiene que pensar desde ya en una solución. Asimismo, La recolección domiciliaria al año fue de 25.118,666 y la recolección de barrido fue de $1.851,840$. De acuerdo con el Plan de Gestión Integral de Residuos Sólidos del Distrito de Barranquilla, No se cuenta con el dato de residuos aprovechados en el último año.

\subsection{Normativa y Planes de Gestión en la ciudad de Madrid}

Asimismo Madrid cuenta con la Ordenanza de Limpieza de los Espacios Públicos y Gestión de Residuos (OLEPGR), con fecha de disposición del 27 de febrero de 2009. La ordenanza tiene por objeto la regulación, en el ámbito de las competencias del Ayuntamiento de Madrid, de todas aquellas conductas y actividades dirigidas a la limpieza de los espacios públicos y privados, así como la recogida, almacenamiento, transporte, valorización, tratamiento y eliminación de los residuos urbanos o municipales, con el fin de conseguir las condiciones adecuadas de salubridad, bienestar ciudadano, pulcritud y ornato, en orden a la debida protección de la salud de las personas, los espacios públicos y el paisaje urbano, así como del medio ambiente, fomentando actitudes encaminadas a mantener la ciudad limpia y posibilitar la reducción, reutilización, reciclado y otras formas de valorización de los residuos. La OLEPGR, especifica 14 Leyes, 7 Reales Decretos, 1 (Una) Orden y algunas directivas de la Unión Europea destacables.

Citado anteriormente el Ayuntamiento de Madrid, dispone de una Ordenanza de Limpieza de los Espacios Públicos y Gestión de Residuos con fecha de Disposición del 27 de febrero de 2009. A efectos de la presente Ordenanza, los residuos urbanos o municipales se clasifican en generales y especiales. Los residuos generales son 
aquellos generados en los domicilios particulares, comercios, oficinas y servicios de la ciudad tales como la limpieza viaria, espacios verdes, áreas recreativas, mercadillos, festejos y actos públicos. Incluye también todos aquellos residuos que no tengan la calificación de peligrosos, y que por su naturaleza y composición puedan asimilarse a los producidos en los anteriores lugares o actividades. Los residuos especiales son aquellos no incluidos en el apartado anterior, cuya composición, cantidad y naturaleza dificultan la segregación, manipulación o valorización convencionales, exigiendo una gestión diferenciada con el fin de no comprometer la recuperación de otras fracciones o evitar un riesgo para el medio ambiente o la salud de las personas.

La competencia del servicio de recogida y transporte de residuos urbanos es competencia del Ayuntamiento de Madrid y será prestado por el servicio Municipal correspondiente con la frecuencia y horario adecuados, dando la publicidad necesaria para conocimiento de la sociedad. En la actualidad, los servicios de recogida y transporte de residuos urbanos o municipales de la ciudad de Madrid, que gestiona la Dirección General de Gestión Ambiental Urbana, son realizados por empresas concesionarias que ejecutan contratos plurianuales de gestión de servicio público, de acuerdo con los requisitos establecidos por los servicios técnicos del Área de Gobierno de Medio Ambiente y Movilidad tanto en los pliegos de condiciones del concurso público como en las labores de control de los mismos que los servicios municipales realizan diariamente.Adicionalmente, en el año 2013 fue aprobado, el nuevo contrato de gestión de residuos sólidos urbanos convirtiendo a Madrid en pionera de este modelo de gestión, que además permitirá ahorrar a las arcas municipales 11 millones de euros. El contrato integra servicios que, a día de hoy, se prestan a través de trece contratos. Consciente de ello, el Ayuntamiento de Madrid ha implantado un sistema que facilita la separación en origen al ciudadano, con el fin de aumentar la cantidad y calidad de los residuos recogidos, lo que redunda en una mayor tasa de reciclaje. Para lograr una recogida selectiva de mayor calidad, en la ciudad, debido a las características climatológicas y urbanísticas, se ha considerado más apropiado una contenerización o envasado de los residuos urbanos más habituales (envases, resto, papel-cartón, vidrio) previo a la recogida propiamente dicha, frente a las soluciones adoptadas en otras ciudades, donde los residuos no se envasan. Así, la recogida y transporte se basa en el uso de camiones compactadores en los cuales se vuelca automáticamente el contenido de los contenedores. El tipo de contenedores y camiones empleados en cada zona se adapta no sólo al diseño urbanístico, sino al entorno cultural y la actividad económica. Además, en Madrid se dispone de otros sistemas de recogida selectiva para residuos peligrosos o especiales (puntos limpios). Que más adelante se expondrán.

Asimismo, a efectos de la Ordenanza, los recipientes para recogida se clasifican en: "Contenedores normalizados de pequeña capacidad (hasta 330 litros). Contenedores normalizados de mayor capacidad (a partir de 700 litros, incluidos los contenedores de carga lateral) que el Ayuntamiento instala en las vías públicas para uso del vecindario. Contenedores homologados tipo columna, instalados en la vía pública para la recogida de papel-cartón y vidrio". Los recipientes normalizados señalados en 
el apartado anterior quedarán identificados de la siguiente forma, en base al tipo de residuos que recepcionen:"Color amarillo para el caso de la fracción de envases ligeros.Color azul para la fracción papel-cartón.Color verde para la fracción vidrio.Cuerpo gris y tapa naranja para la fracción resto".

Por otra parte, La producción ocasional de residuos, seria en el supuesto que alguien tuviera que desprenderse de residuos urbanos de características especiales o en cantidades superiores a las que constituyen su producción diaria normal, no podrá presentarlos conjuntamente con los residuos habituales. En tal caso, tendrá que contar con autorización para la gestión de los residuos urbanos con sus propios medios a los puntos de transformación o eliminación que indique el servicio Municipal competente, o bien podrá solicitar su retirada al mencionado Servicio. Otros residuos especiales, como residuos de poda y jardinería derivados de operaciones de desbroce de hierbas, siegas, podas y talas (restos vegetales) no podrán depositarse en los contenedores ubicados en la vía pública para la fracción restos. Si la cantidad generada es inferior a 240 litros, podrán depositarse en un Punto Limpio. Por otro lado, los puntos limpios son instalaciones o vehículos de titularidad municipal destinados a la recogida selectiva de residuos urbanos de origen doméstico en los que el usuario deposita los residuos segregados para facilitar su valorización o eliminación posterior. El Ayuntamiento de Madrid tiene dos modelos de Puntos Limpios: Fijos y Móviles, El Punto Limpio Fijo es un centro municipal de transferencia intermedia para la recogida selectiva de determinados residuos, de acceso público y utilizado por el vecindario del municipio y los Puntos Limpios Móviles son vehículos especialmente diseñados para hacer las funciones de recogida de ciertos residuos que el vecindario de la ciudad a menudo produce y que no deben ser mezclados con los que se recogen a diario y que habitualmente generan en sus domicilios. En los Puntos Limpios, tanto Fijos como Móviles, sólo se admitirán, de forma general, residuos generados por domicilios particulares.. Por otra parte, la limpieza urbana es clave en la calidad del espacio público. La ciudad de Madrid tiene una dinámica poblacional compleja ya que, al elevado número de habitantes censados que viven en ella, hay que añadir numerosos visitantes por motivos de ocio o trabajo. Esta realidad hace que el servicio público de limpieza sea más complejo que en otros espacios urbanos desde el punto de vista de su frecuencia y especialización.

Por otro lado, En Madrid hay registrados más de 300.000 perros, con lo que esto puede suponer en excrementos en las calles. En la Ordenanza de Limpieza de los Espacios Públicos y Gestión de Residuos, está regulada la obligación de los propietarios de recoger los excrementos de sus mascotas, conducta cuyo incumplimiento es sancionable. En la actualidad, hay instaladas en la ciudad de Madrid 6.000 papeleras del modelo Cibeles que cuentan con expendedores de bolsas biodegradables.La limpieza del espacio público se basa en la combinación de diferentes prestaciones o técnicas, entre ellas barridos y baldeos, ya sean manuales, mecánicos o mixtos, que se repiten cuando es preciso, ajustándolas a las necesidades de la ciudad en cada momento. Por otra parte y mencionado el SELUR es un servicio especial de limpieza urgente e integral, diseñado para atender de forma inmediata y con los medios adecuados, aquellas situaciones de carácter excepcional que puedan 
producirse, tales como incidencias climatológicas, catástrofes naturales, accidentes de cualquier naturaleza, incendios, actos terroristas, limpiezas por motivos específicos de salubridad e higiene, limpieza de manifestaciones y eventos y cualquier otra incidencia especial que pueda requerir la actuación de un servicio de limpieza integral especialmente preparado, lo antes posible y con el mínimo trastorno para el ciudadano.El segundo paso del proceso es la recogida y transporte de estos residuos, generalmente mediante camiones recolectores compactadores, que los trasladan hasta las plantas de tratamiento y recuperación de que dispone el Ayuntamiento de Madrid en el complejo de Valdemingómez, o hasta plantas específicas de reciclado para determinados residuos.

El tercer y último paso corresponde al tratamiento, valorización y eliminación de los residuos, con recuperación de las fracciones susceptibles de serlo. La mayor parte de los residuos que se recogen en la ciudad son posteriormente tratados en las plantas del Parque Tecnológico de Valdemingómez, donde se obtienen materiales reciclables y fuentes de energía. Todas las actividades de esta tercera fase que se llevan a cabo en las plantas municipales, son gestionadas por la Dirección General del Parque Tecnológico de Valdemingómez.

Este Parque se concentra desde 1978 todas las instalaciones de tratamiento de residuos urbanos de Madrid, a las que llegan las más de cuatro mil toneladas que se generan a diario en la cuidad. La concepción del Parque Tecnológico de Valdemingómez y su desarrollo fueron diseñados 9 años antes de que entrara en vigor la Directiva Europea 2008/98/CE de 19 de noviembre, que obliga a recuperar todos los materiales y energía contenidos en los residuos, criterio de trabajo que el Parque Tecnológico de Valdemingómez ha sido pionero en incorporar. Su objetivo esencial es procesar los residuos para aprovechar todo lo que se pueda recuperar de ellos y depositar los residuos no recuperables de forma segura en un vertedero. Para cumplir este objetivo, sus centros cuentan con una amplia gama de instalaciones con distintas funciones. Estos centros son Las Lomas, La Paloma, Las Dehesas, y La Galiana, el complejo de Biometanización, además del Centro de Visitantes y cinco instalaciones educativas.

El Centro La Paloma, Su función es recuperar los materiales reciclables presentes en los residuos de las bolsas de envases y restos, y separar y procesar la materia orgánica para transformarla en compost. Asimismo el Centro Las Lomas, perteneciente al Parque Tecnológico de Valdemingómez, reúne en una única instalación los procesos de recuperación de materiales reciclables, compostaje y reciclaje energético.El Centro La Galiana, Sus funciones principales son el aprovechamiento energético del biogás generado en el antiguo vertedero de Valdemingómez (1978-2000), y la conservación del Parque Forestal instalado sobre este último.

El Centro La Galiana es resultado de un proyecto municipal denominado Desgasificación con recuperación energética, sellado, control y mantenimiento del vertedero de Valdemingómez, finalizado en 2003. El Centro Las Dehesas es un modelo de equilibrio entre actividad productiva y respeto al medio ambiente. Los 
principales procesos que se desarrollan en este Centro, inaugurado en el año 2000, son la separación y clasificación de materiales reciclables y el compostaje de la materia orgánica, la incineración de animales muertos, el depósito en vertedero, este último acompañado de la extracción de biogás para su futuro aprovechamiento energético, el tratamiento de residuos voluminosos y una planta de granceado y extrusión de PEAD.El Complejo de Biometanización. La biometanización es un proceso biológico de digestión anaerobia, cuyo objetivo es transformar la fracción orgánica seleccionada en las plantas de separación en dos productos: biogás y digesto. El biogás es una valiosa fuente de energía, mientras que el digesto constituye una excelente materia prima para la fabricación de abono (compost).

Aunque la población de Madrid ha crecido, en los últimos diez años, la población permanece estable. Entre los años 2004 y 2014 lo máximo que alcanzo fue de 3.284.110 habitantes equivalente al año 2010. Desde el año 2008, la cantidad de residuos generados por la ciudad ha disminuido. El ciudadano de Madrid, no sólo ha ido generando menos residuos, sino que, además, realiza cada vez mejor la separación selectiva en origen de sus residuos, en otras palabras, separa cada vez mejor en casa sus residuos. Esto y el incremento de eficacia en el tratamiento de residuos, ha ayudado a que la recuperación de los materiales reciclables haya aumentado de manera significativa.

La población generadora equivalentees de3.709.697. La población empadronada es de 3.165.235. Las toneladas anuales generadas son de 1.219.623. Las toneladas generadas diariamente son de 3.341. La tasa de generación por habitante empadronado y año ( $\mathrm{kg}$ hab/año) es de 385,20. Y la tasa de generación diaria por habitante empadronado ( $\mathrm{kg}$ hab/día) es de 1,06. El reciclaje de materiales recuperados en la planta son de 71.082 toneladas, distribuidos de la siguiente manera: 17.938 toneladas de plásticos. 16.172 toneladas de ferromagnéticos. 4.525 toneladas de férrico quemado. 886 toneladas de aluminio. 893 toneladas de otros metales. 24.560 toneladas de papel y cartón. 2.655 toneladas de brik. 700 toneladas de vidrio. 14.462 toneladas de compost producido. Asimismo, el reciclaje energético recuperado fue de 207.013 MWh (megavatio-hora) de energía eléctrica, procedentes de la valorización energética de los rechazos de los procesos de separación y clasificación, así como el aprovechamiento como combustible del biogás generado en el antiguo vertedero de Valdemingómez.El $76.7 \%$ de esta cantidad (158.814 MWh) se exportó a la red, yel $23.3 \%$ restante $(48.200 \mathrm{MWh})$ se consumió en los propios Centros. Por otro lado el número de visitantes dentro del Programa Integral de Educación Ambiental del Parque Tecnológico de Valdemingómez es de 8.203.Finalmente y una vez clasificados en estas instalaciones, los envases son posteriormente puestos a disposición a los recicladores, quienes los transformarán en nueva materia prima, cerrando con ello el ciclo del envase y dando así una nueva vida a los materiales.En este sentido, se realizan procedimientos de adjudicación de materiales procedentes de plantas de clasificación de envases, solicitud de homologación para las empresas interesadas. 


\section{Conclusión}

El análisis comparativo entre Madrid y el distrito de Barranquilla, ha puesto de manifiesto grandes diferencias tanto en lo que se refiere a la educación ambiental de los ciudadanos en el tema de los RSU como en la normativa que regula el tratamiento y la gestión de los mismos. Tomando como modelo la ciudad de Madrid, el municipio colombiano tendría que adoptar normas específicamente orientadas a la gestión de los RSU. Dichas normas, deberían permitir el establecimiento de objetivos a corto y largo plazo cuyo incumplimiento por parte de las administraciones o ciudadanos suponga algún tipo de sanción. Sería fundamental adoptar algunas medidas que faciliten la separación de los materiales de desecho en origen y, por supuesto, se recomienda incidir de manera más efectiva en el tema a través de una buena y planificada estrategia de educación ambiental. La conexión entre normas, procesos de gestión y educación ambiental parece ser una vía de éxito para lograr ciudades limpias y energéticamente eficaces.

\section{Bibliografía}

Alcaldía de Barranquilla (2016): Información general. Consulta: 03 de mayo de 2016. http://www.barranquilla.gov.co/

Ayuntamiento de Madrid (2009): ANM 2009\6. Ordenanza de Limpieza de los Espacios Públicos y Gestión de Residuos. Madrid, 27 de febrero.

https://sede.madrid.es/framework/generacionpdf/anm2009_6.pdf?idnormativa=4bde9faac 2330210vgnvem2000000c205a0arcrd\&nombrefichero=anm2009_6\&cachekey $=7$

Departamento Administrativo Nacional de Estadística (DANE) (2016): Información general. Consulta: 05 de marzo de 2016.Disponible en: www.dane.gov.co

El Heraldo., 2011-2016 (2016): Periódico El Heraldo de Barranquilla. El Heraldo. Barranquilla. Consulta: 04 de mayo de 2016.

http://www.elheraldo.co/local/el-fantasma-de-la-liquidacion-ronda-al-damab-250932

Herrera, A. (2016): Vive Barranquilla Limpia. Correo electrónico del 17 de julio a Ana Herrera. [Informe]. P 1-11.

Instituto Nacional de Estadística (INE), 2015-2016. Consulta: 02 de marzo de 2016. Disponible en:http://www.ine.es/dynt3/inebase/es/index.html?padre $=517 \& \mathrm{dh}=1$

Ministerio de Medio Ambiente (1997): Educación ambiental para el desarrollo sostenible. Seminario permanente sobre evaluación de programas de educación ambiental. Tomo 4. Madrid: Centro de publicaciones. Secretaria General Técnica. Ministerio de Medio Ambiente. P 15-16.

Romano, D., Barrenechea, P. (2011): Instrumentos económicos para la prevención y el reciclaje de los residuos urbanos. Zaragoza. Fundación Ecológica y Desarrollo.

Secretaria de Planeación (2015): Plan de Gestión Integral de Residuos Sólidos-PGIRS 20162027. file://c:/users/hp\%201000/downloads/pgirs\%20distrito\%20barranquilla\%2020162027\%20definitivo\%20(6).pdf 